\title{
Author Correction: Experimental extraction of the quantum effective action for a non-equilibrium many-body system
}

Maximilian Prüfer (D), Torsten V. Zache DD, Philipp Kunkel, Stefan Lannig, Alexis Bonnin, Helmut Strobel, Jürgen Berges and Markus K. Oberthaler (D)

Correction to: Nature Physics https://doi.org/10.1038/s41567-020-0933-6, published online 15 June 2020.

In the version of this Letter originally published online, in the Methods subsection 'Explicit expressions for correlators', the citation of equation (9a) was incorrect; it should have been a citation of equation (8a). Also, in equation (8c), on the right-hand side the final term was given as $+2 \mathrm{O}_{a} O_{b} O_{c}$; it should have been $-O_{a} O_{b} O_{c}$. In equation (8d), the final seven terms on the right-hand side were given as $-6 O_{a} O_{b} O_{c} O_{d}+2 C_{a b} O_{c} O_{d}+2 C_{a c} O_{d} O_{b}+2 C_{a d} O_{b} O_{c}+2 C_{b c} O_{d} O_{a}+2 C_{b d} O_{a} O_{c}+2 C_{c d} O_{a} O_{b}$, but should have been $-O_{a} O_{b} O_{c} O_{d}-C_{a b} O_{c} O_{d}-$ $C_{a c} O_{d} O_{b}-C_{a d} O_{b} O_{c}-C_{b c} O_{d} O_{a}-C_{b d} O_{a} O_{c}-C_{c d} O_{a} O_{b}$. All versions of the Letter have been amended.

Published online: 12 August 2020

https://doi.org/10.1038/s41567-020-1024-4

(c) The Author(s), under exclusive licence to Springer Nature Limited 2020

\section{Publisher Correction: Ultrasound evidence for a two-component superconducting order parameter in $\mathrm{Sr}_{2} \mathrm{RuO}_{4}$}

S. Benhabib, C. Lupien (D), I. Paul, L. Berges, M. Dion D, M. Nardone, A. Zitouni, Z. Q. Mao (D), Y. Maeno iD, A. Georges (D),

L. Taillefer (iD and C. Proust (iD

Correction to: Nature Physics https://doi.org/10.1038/s41567-020-1033-3, published online 21 September 2020.

In the PDF version of this Letter originally published, the equation for the cross-coupling term, $F_{\Delta-u}$, was incorrect: extraneous LaTeX code, '0.5em', was included and part of the final term and closing parenthesis, 'c.c.)', was missing. The HTML version was correct. The PDF version of the Letter has now been corrected.

Published online: 28 October 2020

https://doi.org/10.1038/s41567-020-01090-2

(C) The Author(s), under exclusive licence to Springer Nature Limited 2020

\section{Publisher Correction: Interacting topological edge channels}

Jonas Strunz Di , Jonas Wiedenmann, Christoph Fleckenstein, Lukas Lunczer, Wouter Beugeling, Valentin L. Müller, Pragya Shekhar, Niccolò Traverso Ziani, Saquib Shamim (D), Johannes Kleinlein (D), Hartmut Buhmann, Björn Trauzettel and Laurens W. Molenkamp

Correction to: Nature Physics https://doi.org/10.1038/s41567-019-0692-4, published online 28 October 2019.

In the version of this Article originally published, in the paragraph beginning 'The bias and temperature dependence', the units for two of the energy ranges of the spin gap were incorrect. ' $\Delta E \approx 150-300 \mathrm{eV}^{\prime}$ should have been ' $\Delta E \approx 150-300 \mu \mathrm{eV}$ ' and ' $\mathrm{eV} / 200-400 \mathrm{eV}$ ' should have been ' $e V_{\mathrm{dc}} \approx 200-400 \mu \mathrm{eV}$ '. The online versions of the Article have been corrected.

Published online: 4 November 2020

https://doi.org/10.1038/s41567-020-01091-1

(c) The Author(s), under exclusive licence to Springer Nature Limited 2020 